\title{
Application of Digital Signal Processing Technology in Electronic Measuring Instruments
}

Gao Xin

China Electronics Technology Group Corporation, the forty - one Institute of Qingdao, Shandong 266500, China

Abstract: With the development of society, digital signal processing technology has been widely used by more and more people to various industries. It is to promote our entire world of information technology development is very favorable, and it can also be the maximum to help people improve the efficiency of working life, so the need to further strengthen its research. In the present situation, digital signal processing technology is the use of electronic equipment to carry out the measurement of the important components of the work, so in the future development process, the need to continue to strengthen the application of digital signal technology for human production and life to provide more the convenience. Based on this paper, the application of digital signal processing technology in the field of electronic measuring instruments is analyzed.

Key words: digital signal processing technology; electronic measuring instruments; application

\section{The concept and characteristics of digital signal processing}

\subsection{Concept}

For digital signal processing, it refers to the use of a variety of converters to people's real life in a variety of real physical information into a certain number, and then deposit to the computer, so that people on the computer can search a variety of information about things ${ }^{1}$. For example, people can be real life in the picture, sound or video are converted into digital information, and then pass these digital signals to people, and this technology in our country are very common in a variety of industries, it belongs to the current line Industry) 'a new application of a new technology, its application can be said to improve the development of our country all walks of life speed. In this paper, we will be for communication, image graphics, instrumentation, PC, hearing aids and automotive electronics systems and other areas of digital signal technology applications to do a specific analysis.

\subsection{Features}

For digital signal processing technology, the first thing is that it is the full use of network technology to deal with information of a work model, it is for our country all walks of life work has played a significant role. And with the development of the times, network technology has been deep into each household, you can say that the computer has become a must for people to produce life, then the digital signal processing technology development prospects are obvious, is very broad. Digital signal processing technology with efficient, fast, cost savings and time, these technologies are not replaced by other products ${ }^{2}$. At the same time, the biggest feature of the digital signal is that it is particularly accurate information processing, which can let people understand a comprehensive and extensive information technology, for people's production and life is very favorable.

\section{Digital signal processing technology in the field of electronic measuring instruments}

\subsection{In the application of the generator}

The generator is a signal source, which is a typical device that provides a variety of frequencies, waveforms and output levels. In measuring the amplitude characteristics, frequency characteristics, transmission characteristics and other electrical parameters of various telecommunication systems or telecommunication equipment, it can be used as a signal source or excitation source for measurement. 
Generator is used in the measurement of frequency synthesis technology, the need to use low-pass filter to complete the corresponding measurement work, and digital signal processing technology is the main content of the filter, the use of digital computing methods, the signal post-processing theory and technology, it can overcome the crystal oscillator can only provide a specific frequency of the corresponding defects, improve the signal output frequency accuracy and stability.

\subsection{In the application of voltage measurement}

Electronic measurement refers to a measurement technology based on electronic technology. It is the product of measurement and electronics.

In the process of electronic measurement, the voltage is a basic parameter of electronic technology measurement, voltage measurement is the basis of electronic measurement. The current voltage measurement has been digitized, the actual application, in the process of digital signal processing in two forms ${ }^{3}$ : First, AC voltage and DC voltage conversion after $\mathrm{A} / \mathrm{D}$ conversion, the other is in the time of the DC voltage Direct $\mathrm{A} / \mathrm{D}$ conversion. In these two conversion methods are related to the $\mathrm{A} / \mathrm{D}$ conversion, $\mathrm{A} / \mathrm{D}$ refers to the analog-to-digital conversion, digital signal processing technology is a very basic part of the analog or continuous changes in the amount of quantification (discretization) converted to the corresponding digital circuit. The A/D conversion consists of three parts: sampling, quantization and coding. If the three parts of the A/D transformation are applied to the voltmeter, it will greatly improve the accuracy of the voltmeter measurement, anti-jamming capability and resolving power.

\subsection{In the application of the oscilloscope}

The oscilloscope is a very versatile electronic measuring instrument that transforms invisible electrical signals into visible images, facilitating the study of changes in electrical phenomena. It is through the form of the waveform to be measured to display the signal, the measured signal through the electron beam hit the screen, the display of the image helps to measure the analysis and interpretation of people, current, voltage and frequency through the oscilloscope. In addition, some of the more special signals can also be displayed on the oscilloscope, such as amplitude modulation, phase difference display often appear in the application range of the oscilloscope.
3. The current digital signal processing technology, the historical origins and forward direction

\subsection{Digital signal processing technology development history}

The development of digital signal processing technology has gone through the basic theory of popular, accepted by consumers and widely used, the level of application of the crowd reached the peak, digital signal processing technology period of these four periods, before the digital signal processing technology, can only use microprocessing, the MPU processing efficiency and speed are relatively low, cannot be achieved to deal with digital signal high speed, high aging requirements ${ }^{4}$. Therefore, the processing speed is faster and more efficient digital signal processing technology, and gradually developed, with the emergence of integrated circuits, development and a wide range of applications, digital signal processing technology, the corresponding vigorous development. Digital signal processing chip generation, creating a new era of digital signal processing technology development. Since then, digital signal processing technology to achieve a leap-style development, and gradually to the miniaturization of the change. With the emergence and development of CMOS technology, the second generation of digital signal processing technology chip, combined with this CMOS technology, greatly improving the storage capacity and computing speed. With the continuous development of science and technology, the fifth generation of digital signal processing devices, also produced accordingly, and will continue to develop, applied to various fields.

\subsection{The current digital signal processing technology development direction}

Digital signal processing technology is still in the initial stage of application, constantly updated digital signal processor, will be applied to a wider range of areas 5 . According to its characteristics and advantages and the current application of the field of view, digital signal processing technology in the future development is mainly reflected in four aspects, the first digital signal processing technology, in the future will pay more attention to improve the speed of operation, reduce the electronic equipment function And the loss of resources, reducing the size of the geometry, making digital signal processing technology to meet the modern society and industrial production in the growing variety of needs. Second, the development of digital signal processing 
technology will focus on the kernel structure and other aspects of some improvement and change, pay more attention to the application of digital signal processor core micro-system structure.

In short, the digital signal processing technology for the measurement of electronic equipment has a very important significance, we must attach importance to digital signal processing technology, need to focus on strengthening its research.

\section{References}

[1] Smith, S., 2013. Digital signal processing: a practical guide for engineers and scientists. Newnes.

[2] Sun Bing. On the Application of Digital Signal Processing Technology and Development Research [J]. Information and Communication, 2015,07: 177.
[3] Yu Jin. Digital Signal Processing Technology Application and Development [J]. Digital Technology and Applications, 2015,12: 224.

[4] Hu Zhizhe. Analysis of Digital Signal Processing Technology Development and Thinking [J]. Henan Science and Technology, 2013,20: 2.

[5] Gupta, V., Mohapatra, D., Raghunathan, A. and Roy, K., 2013. Low-power digital signal processing using approximate adders. IEEE Transactions on Computer-Aided Design of Integrated Circuits and Systems, 32(1), pp.124-137. 